\title{
Strong T-cell costimulation can reactivate tumor antigen-specific $T$ cells in late-stage metastasized colorectal carcinoma patients: Results from a phase I clinical study
}

\author{
VOLKER SCHIRRMACHER, CHRISTOPH SCHLUDE, JÜRGEN WEITZ and PHILIPP BECKHOVE \\ German Cancer Research Center, Division of Translational Immunology; Department of \\ Surgical Oncology, Heidelberg University Hospital, Heidelberg, Germany
}

Received August 1, 2014; Accepted September 6, 2014

DOI: $10.3892 /$ ijo.2014.2692

\begin{abstract}
T-cell costimulation is necessary to induce a response of naïve $\mathrm{T}$ cells. Whether T-cell costimulation can also cause reactivation of unreactive, possibly anergized memory T cells (MTCs) from late-stage cancer patients is unknown. To investigate this question, we developed a bispecific anti-CD28 fusion protein (bsHN-CD28) which can easily be attached to the vaccine ATV-NDV. This virus-modified autologous tumor cell vaccine has already shown effectivity in colon cancer patients following resection of liver metastases. In this phase I clinical study, 14 colorectal carcinoma (CRC) patients with late-stage disease which could not be operated anymore with curative intent were treated with the vaccine ATV-NDV to which bsHN-CD28 was attached. No severe adverse events were recorded. All patients showed an immunological response of tumor-reactive $\mathrm{T}$ cells, at least once during the course of five vaccinations. Also, we demonstrate a dose-response relationship with the costimulatory molecule added to the vaccine. A partial response of metastases was documented in four patients. The study suggests that the three-component vaccine is safe and can reactivate possibly anergized $\mathrm{T}$ cells from a chronic disease like advanced-stage cancer.
\end{abstract}

\section{Introduction}

Colorectal carcinoma (CRC) is the most frequent type of cancer in Germany. Death is mostly due to development of distant metastases in liver and/or lung. Surgical resection of metastases with curative intent is possible only in a small proportion (10-15\%) of patients (1). If this is not possible, such patients normally receive palliative chemotherapy. They die on average within a period of 2 years. The development of new

Correspondence to: Professor Volker Schirrmacher, Present address: IOZK Cologne, Hohenstaufenring 30-32, D-50674 Cologne, Germany

E-mail:v.schirrmacher@web.de

Key words: bispecific antibodies, T-cell costimulation, colorectal carcinoma, ELISPOT response therapeutic strategies is therefore urgently needed for patients with metastasized CRC.

Immunotherapy is a new strategy which looks promising for CRC because this tumor is immunogenic and patients with distinct tumor-infiltrating $\mathrm{T}$ cells have a higher survival probability than those without such immune cells (2). Active vaccination aims at supporting the generation of a polyclonal T-cell response to multiple tumor-associated antigens (TAAs). In the past, we developed a live tumor cell vaccine for CRC and other cancers. This ATV-NDV vaccine is composed of 10 million autologous tumor cells which are first infected by a bird paramyxovirus, Newcastle disease virus (NDV) and then become irradiated with $200 \mathrm{~Gy}$ (3). The advantages of the use of autologous tumor cells and of NDV as adjuvans to introduce so-called danger signals have been described (4). A randomized prospective study revealed a significant long-term survival benefit for colon cancer patients upon vaccination with ATV-NDV following resection of liver metastases (5).

Vaccination of patients in late-stage disease has in general been rather ineffective (6), possibly due to dysfunction of the immune system. Tumor-reactive $\mathrm{T}$ cells from such patients are likely anergized (7) because of long-term confrontation with TAAs in the absence of costimulatory signals (8). We hypothesized that it may perhaps be possible to partially reactivate such $\mathrm{T}$ cells by providing strong costimulatory signals in combination with TAAs. To this end and to further increase the effectivity of the vaccine ATV-NDV, we developed in the past bispecific single-chain fusion proteins (bs-scFvs) based on antibodies directed against NDV and against CD28 (9). The construct bsHN-CD28 binds directly to the ATV-NDV vaccine cells while the second arm is directed against CD28, an important molecule on $\mathrm{T}$ cells to deliver costimulatory signals (10).

This report describes the study design, the production of the vaccine ATV-NDV-bsHN-CD28 and the results. The latter are primarily based on the T-cell responses of patients as analyzed by interferon- $\gamma$ ELISPOT assays.

\section{Materials and methods}

Study design. The study was initiated in 2004 after a positive response from the Ethics Committee of the University of 
Heidelberg (no. L-149/2004). Included were only patients who suffered from CRC UICC stage IV (with distant metastases). Tumor tissue was obtained either from operation specimens or from interventional punction of liver metastases. After generation of the vaccine (see below), the patients received five intracutanous vaccinations in the thigh. The first four vaccines were applied at 2-week intervals and the fifth after 4 weeks. Blood samples were taken regularly immediately before and $72 \mathrm{~h}$ after each vaccination for immunological monitoring of the patients T-cell response (see below).

In this phase I study, the dose of the bispecific fusion protein bsHN-CD28 to be added to the ten million ATV-NDV vaccine cells was stepwise increased according to the Fibonacci scheme: D0, D1, D2, D3 (=Dm). D0=0.199 Dm; $\mathrm{D} 1=0.398 \mathrm{Dm} ; \mathrm{D} 2=0.658 \mathrm{Dm} ; \mathrm{D} 3=\mathrm{Dm}(=1 \mu \mathrm{g}$ protein $)$.

Patient characteristics. Included were 14 patients, five women and nine men, median age 55 years (range, 39-71). The primary tumor was either from rectum $(n=7)$, sigma $(n=3)$, colon $(n=1)$ or coecum $(n=3)$. Metastases were localized in liver $(n=13)$, lung $(n=5)$ or in the peritoneum $(n=4)$. Before inclusion into the study, the tumors were progressive under chemotherapy $(n=6)$, stable under chemotherapy $(n=1)$ or there was progress without chemotherapy of synchronous metastases $(n=7)$.

Generation of the vaccine $A T V-N D V-b s H N-C D 28$. The vaccine was generated in the cell culture laboratory of the Department of Surgical Oncology at the Heidelberg University Hospital. After enzymatic digestion of mechanically dissected tumor samples, the single cells were either used directly for further modification or they were first expanded in primary cell cultures to reach the necessary number of cells (at least 50 million). The cells were then modified by infection with NDV (strain Ulster) as described (3) and the bsHN-CD28 protein added at the required amount.

The construction, production and purification of bsHN-CD28 from the plasmid pERdhfr $(\alpha \mathrm{HN}-\alpha \mathrm{CD} 28)$ was described before (11). Transfection of the plasmids into $\mathrm{dCHO}$ cells was carried out by electroporation. Clones that were stably expressing the desired fusion protein were selected by limiting dilution. The production was conducted in high density cell culture systems (Integra Biosciences AG, Zizers, Switzerland). The E tag containing fusion protein was purified by means of an anti-E tag immunoaffinity chromatography procedure (Amersham Pharmacia Biotech, Amersham, UK). The protein concentrations were determined with the CB-Protein Assay ${ }^{\mathrm{TM}}$ reagent (Calbiochem-Merck Co., Schawalbach, Germany). Optimization studies were performed earlier to find the best protocol for its coupling to viral hemagglutinin-neuraminidase $(\mathrm{HN})$ anchor molecules of the vaccine ATV-NDV (12). Before application, the modified vaccine cells were inactivated by gamma irradiation (200 Gy). In addition, the absence of pathogens and fungi had been confirmed by cultivation on blood-agar or on Sabouraud-agar plates.

ELISPOT assay. The isolation of T cells, the generation of dendritic cells (DCs) and the performance of the ELISPOT assay was done essentially as described before $(13,14)$.

\section{Results}

Construction of bsHN-CD28. Fig. 1 shows a scheme of the construction of NDV-specific T-cell stimulatory fusion proteins. Single-chain (scFv) antibodies specific for NDV HN were derived from the hybridoma HN.B (Dr R.M. Iorio, University of Massachusetts, Worcester, MA, USA). The CD28-specific scFv was derived from the hybridoma 9.3 (Dr J.D. Hansen, US Geological Survey-Western Fisheries Research Center, Seattle, WA, USA). The plasmid that encoded the fusion protein bsHN-CD28 (no. 290) had the structure Flag- $\mathrm{V}_{\mathrm{H}}-\mathrm{L}-\mathrm{V}_{\mathrm{L}}-\mathrm{L}-\mathrm{L}-\mathrm{V}_{\mathrm{H}}-\mathrm{V}_{\mathrm{L}}-\mathrm{E}$ tag. Other plasmids encoded anti-CD3 instead of anti-CD28 and human IL-2 instead of the linker poly-1-lysine (L) (10-12).

The highest dose of bsHN-CD28 that was added to the vaccine ATV-NDV in the patient group D3 $(\mathrm{n}=4)$ was $1 \mu \mathrm{g}$ purified protein. The group D2 $(n=4)$ received $1 / 3$ of this amount, the group D1 $(n=3) 1 / 9$ and the group D0 $(n=3) 1 / 27 \mu \mathrm{g}$.

Side-effects. The vaccinations were well tolerated. Severe adverse events were not observed. At the injection site swellings often occurred with indurations which caused itching. Systemic symptoms were subfebrile temperature $\left(37-38^{\circ} \mathrm{C}\right)$ $(n=8)$, fever $\left(>38^{\circ} \mathrm{C}\right)(n=2)$, vomiting $(n=2)$, tiredness $(n=2)$ and abdominal pain $(n=2)$.

Immune response monitoring. The ELISPOT assay was adjusted to measure selectively the reactivity of memory $\mathrm{T}$ cells (MTCs). Purified T cells from the peripheral blood were co-incubated for $40 \mathrm{~h}$ with antigen-presenting DCs and the number of IFN- $\gamma$ secreting T cells per 75,000 or 100,000 $\mathrm{T}$ cells enumerated. Per patient there were eight time points at which blood probes were taken.

Response to autologous tumor lysate. The vaccinations were performed with viable but irradiated autologous tumor cells whose $\mathrm{T}$ cell costimulatory function had been enhanced in two ways: i) by infection with NDV (15); and ii) by attachment of bsHN-CD28 (10-12). The T cells thereby received very proper costimulatory signals.

As can be seen from Fig. 2, none of the 14 patients showed a response at day 0 , that is before the first vaccination. In contrast, at the seven time points after vaccination, always $\sim 40 \%$ of the patients were responsive. These were not always the same patients whose $\mathrm{T}$ cells responded. When analyzing the response rate of each patient either before vaccination or after vaccination during the whole time period of 3-73 days, $100 \%$ of them showed at least once a response after vaccination. This is remarkable considering the fact that they were all late-stage metastasized patients. This result is significant and based on $\sim 500$ ELISPOTs.

Response to defined tumor antigens. Since no information exists on the relationship between the vaccination with whole autologous tumor cells and the response to defined TAAs we investigated this further.

The patient DCs were pulsed either with autologous tumor lysate (16) or loaded with known 20mer peptides from defined common TAAs as described $(13,14)$ and used for MTC stimulation. Fig. 3 shows results from one patient (no. 1622). As can be seen, there existed a response already before vaccination to the TAAs CEA, Her2/neu, Mage-3 and p53. 


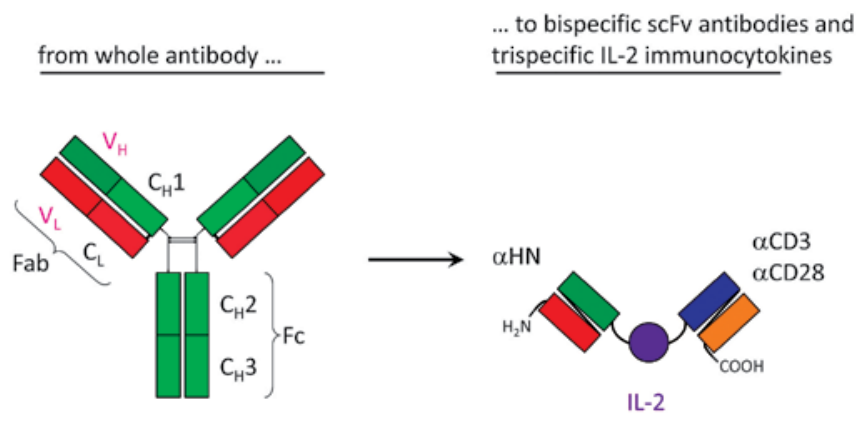

Figure 1. Scheme for the construction of bsHN-CD28 and the related molecules bsHN-CD3 and tsHN-IL-2-CD28. All of these new fusion proteins bind with one arm to the hemagglutinin-neuraminidase (HN) molecule of the Newcastle disease virus (NDV). The second arm is directed to receptors on the surface of T cells, namely CD3 or CD28 and a third ligand (IL-2) was cloned into it to bind to the IL-2 receptor CD25 on T cells, which delivers a further costimulatory signal (10).
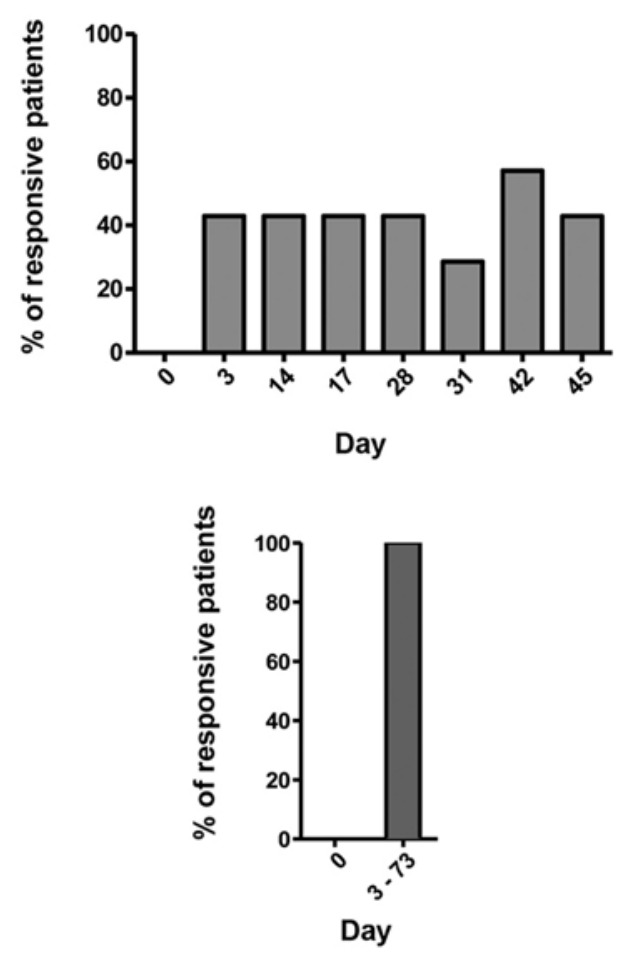

Figure 2. Percentage of responsive colorectal carcinoma (CRC) patients from $\mathrm{n}=14$ to vaccination with ATV-NDV-bsHN-CD28. Responsiveness is defined by interferon- $\gamma$ ELISPOT analysis of T cells from the peripheral blood upon short-term stimulation with autologous dendritic cells (DCs) pulsed with autologous tumor lysate. Before the vaccination, none of the patients showed a response. The upper panel shows the response rate against tumor lysates at each time point after vaccination. The lower panel shows the response rate against tumor lysates of all patients during the whole time period of vaccinations.

This pre-existing memory pool was apparently not sufficient to control the tumor of the patient. Three days after the first vaccination, this patient had responded by a strong expansion of these TAA-specific T cells. This suggests that the vaccine contained all of these common TAAs. Through combination with powerful costimulatory signals the vaccine cells became immunogenic and caused a polyvalent augmentation of TAA-specific T cells. This result corroborates our concept of the design of this vaccine. Also, the number of cells in the

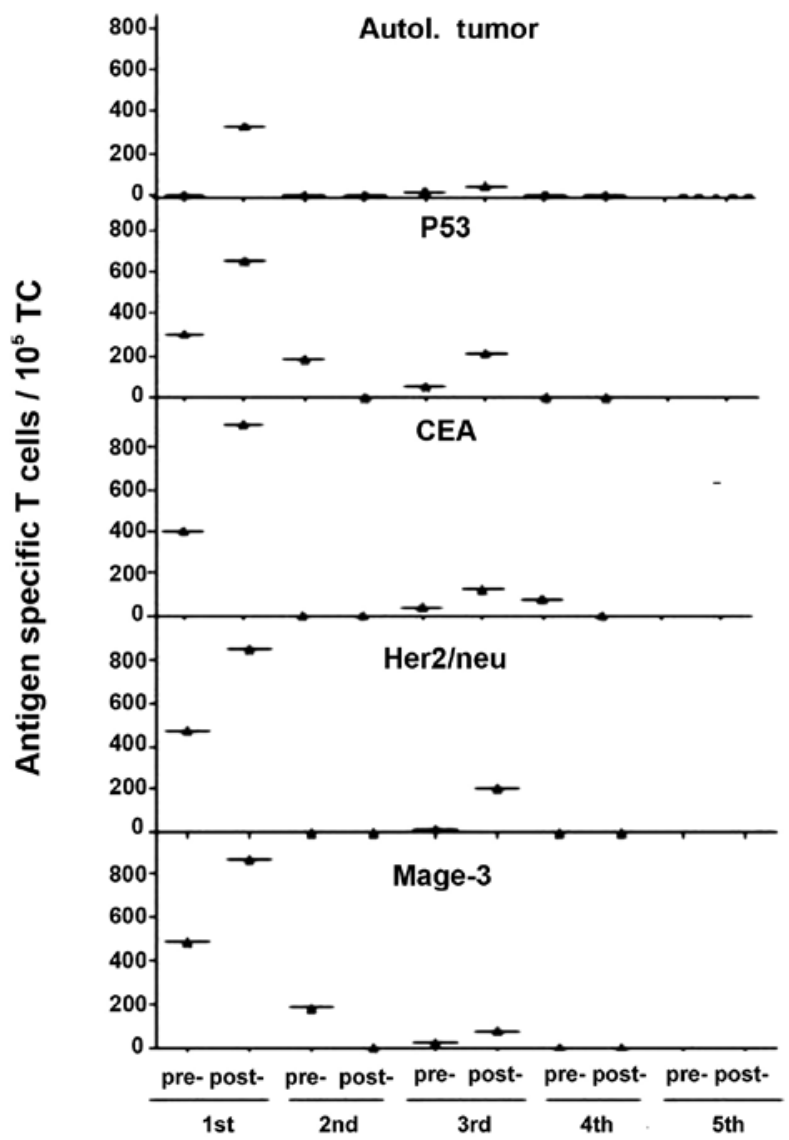

Figure 3. ELISPOT responses to 20mer oligopeptides from defined common tumor-associated antigens (TAAs) in comparison to response to tumor lysate of patient no. 1622 during the course of vaccinations. Most informative is the response measured 3 days after the first vaccination.

vaccine and of other components seems to be physiological and capable of activating a fast MTC response from patients with metastasized CRC.

While in patient no. 1622 the vaccine was immunogenic towards all TAAs used in the ELISPOT assay, the T cells from another patient (no. 1630) showed a dominant response only against Her2/neu and p53 and not to CEA or Mage-3 (data not shown). We showed before for breast cancer patients that the tumor-reactive MTC repertoire is highly individual (14).

Fig. 4 shows results from a correlation analysis of the frequency of patients $\mathrm{T}$ cells responding to autologous tumor lysate and the frequency of TAA peptide-specific T cells. The analysis revealed a significant correlation. This result also means that our protocol of pulsing DCs with tumor lysate leads to presentation of TAA-derived MHC-peptide complexes in similarity to external loading with defined peptides.

Cumulative analysis of TAA peptide-specific $T$ cells. Within the first 3 days after the first vaccination there was in this cumulative analysis of the data from all patients a strong and highly significant $(\mathrm{P}=0.0002)$ increase of TAA peptide-specific $\mathrm{T}$ cells (Fig. 5, left side). Also, the difference before and after all vaccinations (Fig. 5, right side) was highly significant. This suggests a functional reactivation of the pre-existing repertoire $(4,14)$ of tumor-reactive MTCs by this vaccine in spite of the advanced disease stage in these patients. 


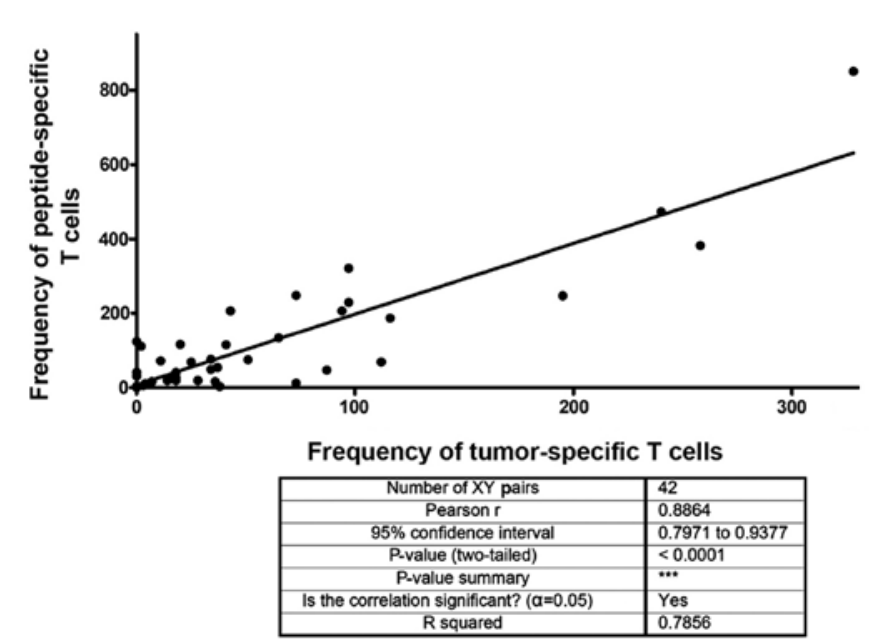

Figure 4. Correlation between responses against dendritic cells (DCs) pulsed with autologous tumor lysate and those to DCs loaded with oligopeptides from the tumor-associated antigen (TAA) Her2/neu. The frequencies of specific $\mathrm{T}$ cells from different patients ( $42 \mathrm{XY}$ pairs) correlate significantly.

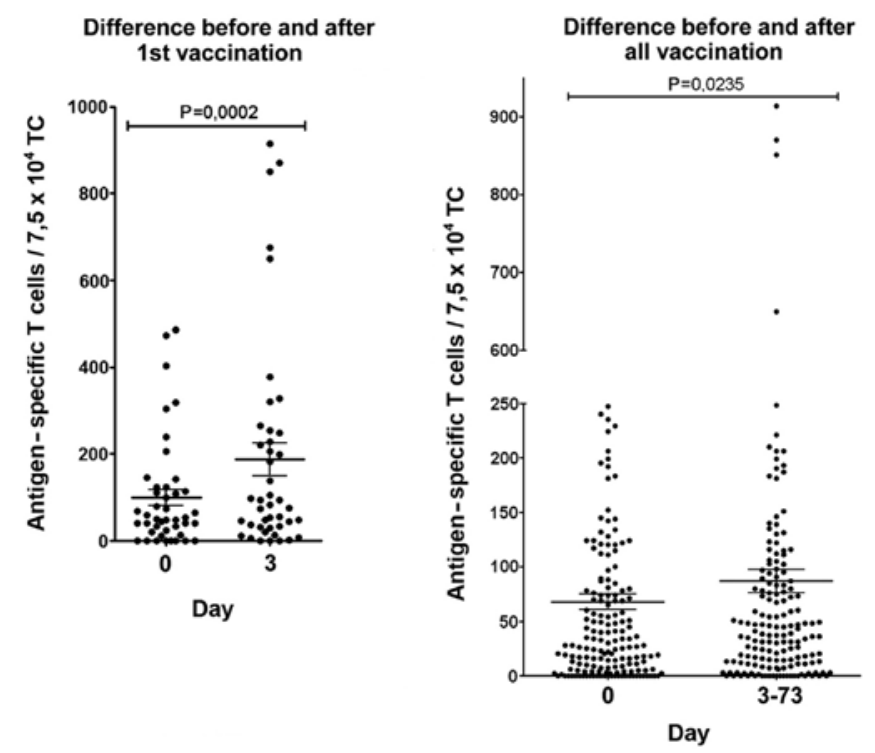

Figure 5. Cumulative analysis of tumor-associated antigen (TAA) peptide specific $\mathrm{T}$ cells from all 14 patients.

Dose-response analysis of bsHN-CD28. An important aspect of the immune response monitoring relates to a possible relationship between the dose of the applied fusion protein in the vaccine and the T-cell response observed. The cumulative analysis of all T-cell responses before and after vaccination, including all the dose groups, had revealed a significant increase of TAA-specific T cells (Fig. 5). The differentiation between the four-dosis groups D0-D3 revealed, however, a clear increase of T-cell responses with increasing dose of the bispecific fusion protein (Fig. 6). This result shows that there was an influence of the added bsHN-CD28 protein on the observed response and corroborates the importance of optimal T-cell costimulation by a tumor vaccine to reactivate pre-existing, perhaps already partially anergic TAA-specific $\mathrm{T}$ cells. That $1 \mu \mathrm{g}$ protein in the vaccine can exert such an effect in late-stage CRC is a remarkable biological activity.

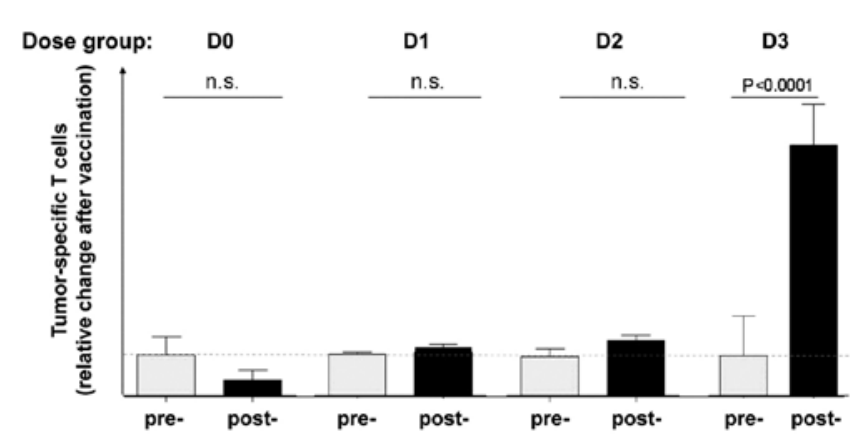

Figure 6. Differential analysis of each dose group of the bsHN-CD28 protein. Means and standard deviations of tumor-associated antigens (TAAs) peptide-specific $\mathrm{T}$ cells before (pre-) and after (post-) the vaccinations. n.s., non-significant.

DTH reactions. The local delayed-type hypersensitivity skin reaction (DTH) was evaluated always 24 and $72 \mathrm{~h}$ after vaccination (3). To distinguish TAA-specific DTH reactions from those against NDV virus or the fusion protein, the skin challenge was performed only with ATV, the inactivated autologous tumor cells. In seven patients an increase of the DTH reaction ( $>100 \%)$ in comparison to the response before vaccination was observed.

Tumor marker. The marker CEA, which was followed during the course of vaccinations, either decreased $(n=4)$, remained where it was $(n=3)$ or it increased $(n=7)$.

Metastases (RECIST criteria). By radiological means the following processes were observed: Partial response $(>30 \%$ decrease) $(n=4)$, stable disease $(n=4)$, progress $(>20 \%$ increase) $(n=5)$. No data exist for one patient who withdrew from the study.

Survival. In 2009, 5 years after initiation of the study, seven of the 14 patients were still alive, two patients had been further operated after a good first response and four patients had died. One patient withdrew from the study.

\section{Discussion}

In this clinical phase I dose escalation study we tested the $\mathrm{T}$-cell costimulatory activity of a bispecific scFv fusion protein, bsHN-CD28. If found to be active in vivo, this new immunostimulatory protein could become attached to any tumor cell infectable by NDV, because the cell surface exposed viral HN molecules would serve as universal anchor molecule for this reagent.

Other bispecific antibodies which are being developed for tumor targeting of immune cells generally use a distinct tumor target structure such as EpCAM (17), HER2 (18), FAP (19) or others. This means that for each tumor type respective reagents need to be developed and tested separately. The advantage of a universal reagent that could be coupled through an adaptor like NDV to any type of tumor appears obvious. Also, this tumor-targeting approach does not interfere with the presentation of TAAs by the tumor cells.

We described that NDV can infect virtually any human tumor cell type and replicate within it, thereby expressing 
at the tumor cell surface a high density of viral $\mathrm{HN}$ and $\mathrm{F}$ molecules (20). Normal cells, in contrast, are resistant to replication of NDV (21). NDV infection of tumor cells was found to introduce a CD80 (B7.1)-independent costimulatory function which was capable of breaking tolerance in vitro (15). It is therefore likely that the vaccine cells in this study can also break tolerance and reverse states of anergy of T cells in situ in cancer patients, especially after augmentation of the costimulatory signals by the bsHN-CD28.

Our concept of introducing into a tumor vaccine a costimulatory function via activation of CD28 on T cells avoids the use of CD28 ligands such as CD80 or CD86. These ligands can also bind to CTLA-4, a receptor mediating negative signals, even with higher affinity. Such interaction would thus downregulate the T-cell response. Instead, we decided to use an agonistic anti-CD28 hybridoma and to clone and select a functional scFv. This was then fused with an anti-HN scFv to create the bispecific product we aimed at.

In preclinical studies the dual binding specificity of the reagent had been demonstrated (11). In further studies, the combination of this reagent with the vaccine ATV-NDV was optimized (10). Once bound, the reagent was stable at the cell surface for a time period of $24-48 \mathrm{~h}$, a time sufficient for T-cell activation (12).

Before discussing the results of this study, it is important to understand how the bsHN-CD28 reagent functions. Both binding sites are monovalent. By itself the reagent can thus not costimulate a $\mathrm{T}$ cell because this requires cross-linking of CD28 molecules at its plasma membrane. Only when multiple bsHN-CD28 molecules become attached to a platform such as the cell surface of the vaccine ATV-NDV they are capable of aggregating CD28 molecules on co-cultured T cells.

Infection of tumor cells by NDV was shown to upregulate expression of MHC and ICAM-1 molecules (22). Similar to professional APCs, the tumor vaccine ATV-NDV-bsHN-CD28 is capable of co-presenting MHC-peptide complexes (including TAAs) to be recognized by the antigen-specific $\mathrm{T}$ cell receptor complex (TCR) to deliver signal 1 and anti-CD28 scFvs to interact with CD28 to deliver signal 2. In addition, ICAM-1 molecules will interact with LFA-1 molecules on T cells thereby stabilizing the cell-cell interaction. It is conceivable that the vaccine cells of our study can form an immunological synapse with T cells, similar to APCs. Such synapse consists of two concentric rings: the central supramolecular activation cluster (c-SMAC) enriched with TCRs and CD28 molecules and the surrounding peripheral supramolecular activation cluster ( $\mathrm{p}-\mathrm{SMAC}$ ) enriched with LFA-1. The $\mathrm{p}-\mathrm{SMAC}$ provides adhesive anchoring of the $\mathrm{T}$ cell to the APC, while the c-SMAC represents a protected zone for sustained signaling via TCR and CD28 (23). It is likely that the strength of positive costimulatory signaling is decisive in a situation of late-stage cancer in which negative signals are likely to dominate.

When signal 2 is lacking, for instance on a TAA-presenting tumor cell, interactions with TAA-specific T cells render the $\mathrm{T}$ cell anergic: the $\mathrm{T}$ cell becomes refractory to signals even when the TCR interacts with TAA. This situation has been described in cancer patients, especially in chronic situations of advanced disease (24), like the patients of this study. T-cell anergy (7) may exist in different states, not all of them being irreversible. For instance, tumor-reactive MTCs from draining lymph nodes of carcinoma patients could be reactivated in a short-term ELISPOT assay using as vaccine ATV-NDV with optimized signals 1 and 2 , but not with a similarly modified vaccine from an unrelated tumor cell line (10).

The main findings of this study are the following:

i) The new fusion protein bsHN-CD28 did not cause major adverse events. A superagonistic anti-CD28 antibody (TGN1412) which has in the past been applied to human beings caused unexpected severe adverse events (25). These were mainly due to non-specific polyclonal T-cell activation and initiation of a so-called cytokine storm reaction. This did not happen with bsHN-CD28. As a monovalent reagent this scFv can only activate $\mathrm{T}$ cells when it is bound on a cell surface to its ligand HN. Even then, it will only activate T cells in combination with signal 1 (10-12). Since NDV is a bird virus, it does not exist in man. Monovalency and dependency on a foreign agent thus explain the high safety profile of this costimulatory molecule.

ii) Vaccination with ATV-NDV-bsHN-CD28 caused a strong significant increase of tumor-reactive $\mathrm{T}$ cells in the peripheral blood of many patients. The fast appearance of tumor-reactive $\mathrm{T}$ cells after the first vaccination is likely due to reactivation and expansion of pre-existing but inactive MTCs. De novo generation of antigen-specific $\mathrm{T}$ cells from the pool of naïve $\mathrm{T}$ cells would require a time period longer than 3 days.

iii) Reactivity of the T cells was directed against DCs pulsed with autologous tumor lysate and also against DCs loaded with peptides from defined common TAAs. Autologous tumor cells express individually distinct (unique) as well as common TAAs and also normal self antigens. The simultaneous analysis of responses to autologous tumor lysate and to peptides from common TAAs revealed similarities, both in terms of frequencies and in terms of kinetics of their appearance in - and disappearance from - the peripheral blood. Responses to autologous tumor lysate correlated with responses to common TAAs. These findings corroborate the assumed presence of TAAs in the vaccine in the form of TAA-derived MHC-peptide complexes. From these data it can be deduced that autologous tumor cells express a broad spectrum of relevant TAAs and that vaccination with modified autologous tumor cells is an effective means to re-enforce a polyvalent TAA-specific repertoire of MTCs (4).

iv) A cumulative analysis of the data from all 14 patients revealed a significant increase of the frequencies of tumor-reactive MTCs in the blood, especially 3 days after the first vaccination. The increase of frequencies of tumor-reactive $\mathrm{T}$ cells suggests expansion of a pre-existing polyclonal pool of TAA-specific MTCs. Some of these may be derived from the bone marrow where they reside long-term in distinct niches (26-28). During the course of multiple vaccinations, the frequencies found in the blood were not always as high as after the first vaccination. This finding could be explained by extravasation of MTCs from the blood into tissues, including tumor tissue. More detailed information on the biology and dynamics of MTC responses in general and about those in cancer patients are required before the phenomena observed can be definitely explained.

v) With increasing dose of the reagent bsHN-CD28 there was a clear-cut increase of tumor-reactive T cells. A dose-response relationship is perhaps the best proof of the 
functionality of a reagent such as the new biological product bsHN-CD28. This seems to be the case in this study. A summary of T-cell analyses from all patients before and after vaccination revealed a significant increase of tumor-reactive $\mathrm{T}$ cells in the whole group (Fig. 5). A differentiation between the different dosis groups revealed nevertheless an increase of T-cell reactivity with increasing dose. The highest and only significant increase was observed with the dose of $1 \mu \mathrm{g}$ protein that was added to 10 million ATV-NDV tumor vaccine cells. This result underlines the importance of the additional costimulation via CD28 and corroborates the assumption that only strong costimulatory signaling can override negative signals on T cells in a chronic disease situation.

vi) All patients showed reactivity to the vaccination, at least once during the time course of immune monitoring. The fact that $100 \%$ of the 14 late-stage cancer patients showed a response, at least once, to this type of vaccine is rather exceptional. Of course, response in this case means T-cell mediated cancer-reactive response which is not equal to clinical response but nevertheless impressive.

With regard to clinical effects, the main observations were decrease of CEA in four patients and partial response of metastases in four patients. Seven patients were still alive in 2009. Unfortunately, we have no further follow-up.

In July 2009, the PI of this study (P.B.) was informed by the Regulatory Office of Karlsruhe (Baden-Württemberg, Germany) that the production of the vaccine for a clinical study is now considered as a somatic cellular therapeutic and that this requires a permit according to legal code no. 13 Arzneim ittelgesetz (AMG). According to study protocol, this phase I clinical study, which generated interesting new information and promising results without having caused severe harm to any patient, should have been followed by a phase II study. Although the study had been approved before, the new regulations via the European Medicines Agency (EMA) do not allow a continuation. The prerequisite for a new study are a GMP status for each of the drug components, tumor cells, NDV and bispecific fusion protein. The time and the costs for this go beyond that what a PI doing research at a public institution can afford. It requires huge investments which only a pharmaceutical company can do. The new regulations are said to be in the interest of the safety of patients but an interest of the pharmaceutical industry in this development can not be disputed. As a consequence of the new legislation, the proportion of PI guided objective non-biased clinical studies among all clinical studies in Europe is dramatically decreasing.

Nevertheless, we hope that the new ideas behing this study will survive the new regulations to the benefit of future cancer patients.

\section{Acknowledgements}

We are gratefull to the Dietmar Hopp Foundation for financial support of this study. It is based on three decades of intensive research by the first author (V.S.) and his team at the German Cancer Research Center, Heidelberg, which paved the way for the concept of active-specific antitumor vaccination using patient-derived tumor cells, infecting them by NDV and finally attaching bispecific antibodies. We thank all the research people, co-operation partners and clinicians involved in the past. With regard to the bispecific antibodies we acknowledge in particular C. Haas, M. Lulei and P. Fournier.

\section{References}

1. Fong Y, Fortner J, Sun RL, Brennan MF and Blumgart LH: Clinical score for predicting recurrence after hepatic resection of metastatic colorectal cancer: analysis of 1001 consecutive cases. Ann Surg 230: 309-318, 1999.

2. Galon J, Costes A, Sanchez-Cabo F, et al: Type, density, and location of immune cells within colorectal tumors predict clinical outcome. Science 313: 1960-1964, 2006.

3. Ockert D, Schirrmacher V, Beck N, et al: Newcastle disease virus-infected intact autologous tumor cell vaccine for adjuvant active specific immunotherapy of resected colorectal carcinoma. Clin Cancer Res 2: 21-28, 1996.

4. Schirrmacher V, Fournier P and Schlag P: Autologous tumor cell vaccines for post-operative active-specific immunotherapy of colorectal carcinoma: long-term patient survival and mechanism of function. Exp Rev Vaccines 13: 117-130, 2014.

5. Schulze T, Kemmner W, Weitz J, et al: Efficiency of adjuvant active specific immunization with Newcastle disease virus modified tumor cells in colorectal cancer patients following resection of liver metastases: results of a prospective randomized trial. Cancer Immunol Immunother 58: 61-69, 2009.

6. Rosenberg SA, Yang JC and Restifo NP: Cancer immunotherapy: moving beyond current vaccines. Nat Med 10: 909-915, 2004.

7. Schwartz RH: T cell anergy. Annu Rev Immunol 21: 305-334, 2003.

8. Greenfield EA, Nguyen KA and Kuchroo VK: CD28/B7 costimulation: a review. Crit Rev Immunol 18: 389-418, 1998.

9. Fournier P and Schirrmacher V: Bispecific antibodies and trispecific immunocytokines for targeting the immune system against cancer: preparing for the future. BioDrugs 27: 35-53, 2013.

10. Aigner M, Janke M, Lulei M, Beckhove P, Fournier P and Schirrmacher V: An effective tumor vaccine optimized for costimulation via bispecific and trispecific fusion proteins. Int $\mathrm{J}$ Oncol 32: 777-789, 2008.

11. Haas C, Lulei M, Fournier P, Arnold A and Schirrmacher V: T-cell triggering by CD3- and CD28-binding molecules linked to a human virus-modified tumor cell vaccine. Vaccine 23 : 2439-2453, 2005.

12. Haas C, Lulei M, Fournier P, Arnold A and Schirrmacher V: A tumor vaccine containing anti-CD3 and anti-CD28 bispecific antibodies triggers strong and durable antitumor activity in human lymphocytes. Int J Cancer 118: 658-667, 2006.

13. Koch M, Beckhove P, Op den Winkel J, et al: Tumor infiltrating T lymphocytes in colorectal cancer: Tumor-selective activation and cytotoxic activity in situ. Ann Surg. 244: 986-992, 2006.

14. Sommerfeldt N, Schütz F, Sohn C, Förster J, Schirrmacher V and Beckhove P: The shaping of a polyvalent and highly individual T-cell repertoire in the bone-marrow of breast cancer patients. Cancer Res 66: 8258-8265, 2006.

15. Termeer CC, Schirrmacher V, Bröcker EB and Becker JC: Newcastle disease virus infection induces B7-1/B7-2-independent T-cell costimulatory activity in human melanoma cells. Cancer Gene Ther 7: 316-323, 2000.

16. Bai L, Koopmann J, Fiola C, Fournier P and Schirrmacher V: Dendritic cells pulsed with viral oncolysates potently stimulate autologous $\mathrm{T}$ cells from cancer patients. Int $\mathrm{J}$ Oncol 21: 685-694, 2002.

17. Brischwein K, Schlereth B, Guller B, et al: MT110: a novel bispecific single-chain antibody construct with high efficacy in eradicating established tumors. Mol Immunol 43: 1129-1143, 2006.

18. Biburger M, Weth R and Wels WS: A novel bispecific tetravalent antibody fusion protein to target costimulatory activity for T-cell activation to tumor cells overexpressing ErbB2/HER2. J Mol Biol 346: 1299-1311, 2005.

19. Müller D, Frey K and Kontermann RE: A novel antibody-4-1BBL fusion protein for targeted costimulation in cancer immunotherapy. J Immunother 31: 714-722, 2008.

20. Schirrmacher V, Haas C, Bonifer R, Ahlert T, Gerhards R and Ertel C: Human tumor cell modification by virus infection: an efficient and safe way to produce cancer vaccine with pleiotropic immune stimulatory properties when using Newcastle disease virus. Gene Ther 6: 63-73, 1999. 
21. Fournier $\mathrm{P}$, Wilden $\mathrm{H}$ and Schirrmacher V: Importance of retinoic acid-inducible gene I and of receptor for Type I interferon for cellular resistance to infection by Newcastle disease virus. Int J Oncol 40: 287-298, 2012.

22. Washburn B and Schirrmacher V: Human tumor cell infection by Newcastle Disease Virus leads to upregulation of HLA and cell adhesion molecules and to induction of interferons, chemokines and finally apoptosis. Int J Oncol 21: 85-93, 2002.

23. Grakoui A, Bromley SK, Sumen C, et al: The immunological synapse: a molecular machine controlling $\mathrm{T}$ cell activation. Science 285: 221-227, 1999.

24. Zitvogel L, Tesniere A and Kroemer G: Cancer despite immunosurveillance: immunoselection and immunosubversion. Nat Rev Immunol 6: 715-727, 2006.

25. St Clair EW: The calm after the cytokine storm: lessons from the TGN1412 trial. J Clin Invest 118: 1344-1347, 2008.
26. Feuerer M, Beckhove P, Bai L, Solomayer EF, Bastert G, Diehl IJ, Pedain C, Oberniedermayr M, Schirrmacher V and Umansky V: Therapy of human tumors in NOD/SCID mice with patient-derived reactivated memory $\mathrm{T}$ cells from bone marrow. Nat Med 7: 452-458, 2001.

27. Feuerer M, Beckhove P, Garbi N, Mahnke Y, Limmer A, Hommel M, Hämmerling GJ, Kyewsky B, Hamann A, Umansly V and Schirrmacher V: Bone marrow as a priming site for T-cell responses to blood-borne antigen. Nat Med 9: 1151-1157, 2003.

28. Schirrmacher V, Feuerer M, Fournier P, Ahlert T, Umansky V and Beckhove P: T-cell priming in bone marrow: the potential for long-lasting protective anti-tumor immunity. Trends Mol Med 9: 526-534, 2003 